\title{
A FIXED POINT THEORY FOR MULTI-VALUED MAPPINGS
}

\author{
LECH PASICKI
}

\begin{abstract}
ABSTRACr. In the present paper several theorems are proved for the multi-valued mappings that operate on the type I and type II spaces defined in [10]. The theorems generalize the well-known results for the locally convex spaces.
\end{abstract}

1. Fundamental theorems. We refer to the Definitions 1-4 from [10].

It is known that every compact self-map of a type I space has a fixed point (see [11]). Now we will prove in a similar way a theorem for the type II spaces.

1.1. TheOREM. Let $X$ be a type II space and let $f: X \rightarrow X$ be a compact map for which $\overline{f(X)}$ is finite dimensional. Then $f$ has a fixed point.

Proof. Let us assume $\operatorname{dim} \overline{f(X)}<p-1$ and Fix $f=\varnothing$.

We can find an open cover $\Psi=\left\{W_{x}\right\}_{x \in \overline{f(X)}}$ of $\overline{f(X)}$ for which $S_{z}\left(I, W_{x}\right) \cap$ $f^{-1}\left(W_{x}\right)=\varnothing, z \in W_{x}$. So we may assume

$$
S_{z_{1}}\left(I, S_{z_{2}}\left(I, \ldots, S_{z_{p}}\left(I, W_{x}\right) \ldots\right) \cap f^{-1}\left(W_{x}\right)=\varnothing\right.
$$

for $z_{1}, \ldots, z_{p} \in W_{x}$ (see [10, (6)]). There exists an open star refinement $\mathcal{Q}$ of order $\leq p$ for $\mathscr{W}\left[3,5.1 .12\right.$, p. $377 ; 7.2 .4$, p. 484]. Choose a finite cover $\left\{U_{i}\right\}_{i=1, \ldots, n}$ of $\overline{f(X)}, \quad x_{i} \in U_{i}$ and $W_{i}:=W_{x(i)} \supset \operatorname{St}\left(U_{i}, \mathcal{Q}\right), i=1, \ldots, n$. Let it be $X_{i}=$ $X \backslash f^{-1}\left(U_{i}\right)=\bar{X}_{i}$. Let us define $g: I^{n} \rightarrow X$ as follows:

$$
g\left(s_{1}, \ldots, s_{n}\right)=S_{x_{1}}\left(t_{1}, S_{x_{2}}\left(t_{2}, \ldots, S_{x_{n-1}}\left(t_{n-1}, x_{n}\right) \ldots\right)\right.
$$

for $\sum_{i=1}^{n} s_{i}=1, t_{i}=s_{i} / \max \left\{s_{i}: i=1, \ldots, n\right\}, i=1, \ldots, n$. Write $K\left\{i_{1}, \ldots, i_{k}\right\}$ $=\left\{g\left(s_{1}, \ldots, s_{n}\right): s_{i}=0\right.$ for $\left.i \neq i_{j}, j=1, \ldots, k\right\}$. Then for $\cap_{j=1}^{k} U_{i j} \neq \varnothing$ (implies $k<p$ ) we have

$$
\begin{aligned}
K\left\{i_{1}, \ldots, i_{k}\right\} & \subset S_{x_{i_{1}}}\left(I, \ldots, S_{x_{i_{k}}}\left(I, W_{i_{1}}\right) \ldots\right) \\
& \subset X \backslash f^{-1}\left(W_{i_{1}}\right) \subset X \backslash \bigcap_{j=1}^{k} f^{-1}\left(U_{i_{j}}\right)=\bigcup_{j=1}^{k} X_{i_{j}},
\end{aligned}
$$

because $x_{i_{1}}, \ldots, x_{i_{k}} \in W_{i_{1}}$. Now it can be easily seen that always $K\left\{i_{1}, \ldots, i_{k}\right\} \subset$ $\cup_{j=1}^{k} X_{i j}$.

The set $g^{-1}\left(K\left\{i_{1}, \ldots, i_{k}\right\}\right)$ contains a $k$-simplex and $g^{-1}\left(X_{i}\right)$ are closed as $g$ is continuous $[3,3.4 .8$, p. 210$]$, (cf. [11]). It can be seen now that $\cap_{i=1}^{n} g^{-1}\left(X_{i}\right) \neq \varnothing$

Received by the editors August 12, 1980.

1980 Mathematics Subject Classification. Primary 54H25, 54C60, 55P99.

Key words and phrases. Homotopy, type I space, fixed point, multi-valued mapping, open cover. 
[3, Theorem 4, p. 510] and hence $\cap_{i=1}^{n} X_{i} \neq \varnothing$. This latter assertion implies

$$
X \neq X \backslash \bigcap_{i=1}^{n} X_{i}=\bigcup_{i=1}^{n} f^{-1}\left(U_{i}\right)=X .
$$

This contradiction proves Fix $f \neq \varnothing$.

In the previous papers of mine $\overline{\cos } A$ was used in place of $\overline{\operatorname{coS} A}$. The next lemma states that $\operatorname{coS} A$ can be treated in another way, similarly as in the linear topological spaces.

1.2. Lemma. Let $X$ be $S$-contractible and for $\varnothing \neq A \subset X$ let us write $\overline{\cos } A=$ $\cap\left\{D=\bar{D} \subset X: S_{x}(I, D) \subset D, x \in A \subset D\right\}$. Then $\overline{\cos } A=\overline{\operatorname{coS} A}$.

Proof. Obviously $\overline{\operatorname{coS} A} \subset \overline{\operatorname{co}} S$. Let it be $D \subset X$ and $S_{x}(I, D) \subset D$ for $x \in A$. Then we have $S_{x}(t, \bar{D}) \subset \overline{S_{x}(t, D)}$ for any $t \in I$ and $x \in A$. Hence

$$
S_{x}(I, \bar{D})=\bigcup_{t \in I} S_{x}(t, \bar{D}) \subset \bigcup_{t \in I} \overline{S_{x}(t, D)} \subset \overline{\bigcup_{t \in I} S_{x}(t, D)} \subset \bar{D}
$$

which gives $\overline{\cos } A \subset \overline{\operatorname{coS} A}$ for $D=\cos A$.

For a space $X$ let $2^{X}, C(X), T(X)$ denote respectively the family of all nonempty, nonempty and closed, nonempty, closed and $S$-convex subsets of $X$.

Suppose $X, Y, Z$ are nonempty and $G: X \rightarrow 2^{Y}$ is a multi-valued mapping. Then for $\varnothing \neq A \subset X G(A):=\cup_{x \in A} G(x)$ and $G(\varnothing)=\varnothing\left[1\right.$, p. 22]. If $G_{1}: Y \rightarrow 2^{Z}$, $\left(G_{1} \circ G\right)(x):=G_{1}(G(x))$ [1, p. 24]. If $H: 2^{Y} \rightarrow 2^{Z}$ is set-to-set function, $(H \circ G)(x)$ $=H(G(x))\left(G(x) \in 2^{Y}\right)$. Let it be in addition $(A \cap G)(x):=A \cap G(x)$ for $A \subset$ $Y$.

1.3. Definition (CP. [1, pp. 114, 116]). Let $X, Y$ be spaces. A multi-valued mapping $G: X \rightarrow 2^{Y}$ is upper semicontinuous if for each neighborhood $V$ of any $G(x)$ there exists a neighborhood $U$ of $x$, for which $G(U) \subset V ; G$ is compact if it is upper semicontinuous and $\overline{G(X)}$ is compact.

In the sequel the multi-valued mappings will be called mappings.

Let us define for an $S$-contractible space $X$ a special set-to-set function as follows: $F(A)=\cap_{U \in \mathscr{Q}_{A}} \overline{\operatorname{coS}} U$, where $\mathscr{U}_{A}$ for $A \in 2^{X}$ are such families
of neighborhoods as satisfy

if $V \in \mathcal{U}_{A}$, there exists $V_{1} \in \mathcal{U}_{A}$ such that for any $\varnothing \neq C \subset V_{1}$ there exists $V_{2} \in \mathcal{U}_{C}, V_{2} \subset V$.

It can be seen that in particular $\bigcup_{A}$ can be the family of all neighborhoods of $A$. Besides, from (4) follows

$$
\varnothing \neq C \subset A \text { implies } F(C) \subset F(A) .
$$

For an $S$-contractible subspace $D=\bar{D}$ of $X F_{\mid 2^{D}}$ will denote the function obtained from $F$ by taking $\vartheta_{A} \cap D$ in place of $\vartheta_{A}$ for $A \in 2^{D}$.

1.4. Theorem. Let $X$ be a normal type I space for which $G: X \rightarrow 2^{X}$ is upper semicontinuous and $\operatorname{coS} G(X)$ is compact. Then $F \circ G$ has a fixed point. 
Proof. Suppose $x \notin(F \circ G)(x)$ for $x \in X$. Thus a neighborhood $V \in \mathcal{Q}_{G(x)}$ and such neighborhood $U$ of $x$ can be found, for which $U \cap \overline{\operatorname{coS}} V=\varnothing$. It follows there exists a neighborhood $P$ of $x$ with $G(P) \subset V_{1} \subset V$ (for $A=G(x) V_{1}, V$ satisfy (4)). In view of (5) we have for $W:=U \cap P, W \cap(F \circ G)(W) \subset U \cap$ $F(G(P))$. We obtain from (4), $U \cap F(G(P)) \subset U \cap F\left(V_{1}\right) \subset U \cap \cos V=\varnothing$. Now it is seen there exists an open cover $\mathscr{W}=\left\{W_{x}\right\}_{x \in \overline{\cos } G(X)}$ of the set $\overline{\cos } G(X)$ satisfying

$$
W_{x} \cap\left(F\left(G\left(W_{x}\right)\right)\right)=\varnothing \text { for } x \in \overline{\cos } G(X) .
$$

It follows $[3,5.1 .12$, p. $377 ; 5.1 .9$, p. 375$]$ that there exists a star finite partition of unity $\mathscr{U}$ subordinated to $\mathscr{W}$. Let us choose from $\mathscr{U}$ a cover $\mathscr{V}=$ $\left\{f_{i}^{-1}(0,1\rangle\right\}_{i=1, \ldots, n}$ of $\overline{\operatorname{coS}} G(X)$. Assume $x_{i} \in V_{i} \in \mathcal{V}, \operatorname{St}\left(V_{i}, \mathcal{V}\right) \subset W_{i} \in \mathcal{W}$ for $i=1, \ldots, n$. In view of Tietze's theorem we may think $f_{i}$ maps $X$ into $I$ for any $i=1, \ldots, n$.

Let us write

$$
\begin{aligned}
& p_{i}(x)=\min \left\{1,\left|1-\sum_{i=1}^{n} f_{i}(x)\right|\right\}, \\
& t_{i}(x)=\left(f_{i}(x)+p_{i}(x)\right) / \max \left\{f_{i}(x)+p_{i}(x): i=1, \ldots, n\right\} .
\end{aligned}
$$

It can be seen that $p_{i}: X \rightarrow I$ are maps, $p_{i}(x) \neq 0$ for $\sum_{i=1}^{n} f_{i}(x)=0$ and $p_{i}(x)=0$ for $x \in \overline{\operatorname{coS}} G(X)$. Besides, for any $x$ there exists an index $i$ for which $t_{i}(x)=1$. Now let it be for $x \in X$ and $y_{i} \in G\left(x_{i}\right)$

$$
h(x)=S_{y_{1}}\left(t_{1}(x), S_{y_{2}}\left(t_{2}(x), \ldots, S_{y_{n-1}}\left(t_{n-1}(x), y_{n}\right) \ldots\right)\right. \text {. }
$$

The continuity of $h$ can be proved in a similar way as the continuity of $g$ (see (2)).

From $h(X) \subset \operatorname{coS} G(X)$ it follows that $h$ has a fixed point. Suppose $x_{0}=h\left(x_{0}\right)$. There exists a neighborhood $W_{i}$ containing

$$
D:=(\overline{\cos } G(X)) \cap \bigcup\left\{f_{i}^{-1}\left((0,1): f_{i}\left(x_{0}\right) \neq 0\right\} \quad\left(x_{0} \in D\right) .\right.
$$

On the other hand $x_{0} \in h(D) \subset \overline{\operatorname{coS}} G\left(W_{i}\right) \subset(F \circ G)\left(W_{i}\right)$ which contradicts (6).

1.5. TheOREM. Let $\overline{\operatorname{coS}} G: X \rightarrow C(X)$ be a compact mapping for a normal type I space $X$. Then $\overline{\operatorname{coS}} \circ G$ has a fixed point.

Proof. Suppose $x \notin \overline{\cos } G(x)$ for $x \in X$. Then there exist two neighborhoods $U, V$ of $x$ and $\cos G(x)$ respectively for which $U \cap V=\varnothing$. The upper semicontinuity of $\overline{\operatorname{coS}} \circ G$ implies the existence of a neighborhood $P$ of $x$ for which $\overline{\operatorname{coS}} G(P) \subset V$. Then for $W_{x}=U \cap P$ we have

$$
W_{x} \cap \overline{\cos } G\left(W_{x}\right) \subset U \cap \overline{\cos } G(P) \subset U \cap V=\varnothing
$$

Now we can take (8) in place of (6) and continue the proof of the previous theorem.

1.6. TheOREM. Let $G: X \rightarrow 2^{X}$ be an upper semicontinuous mapping for a normal space of type II. Then $F \circ G$ has a fixed point if $\overline{c o S} G(X)$ is compact and finite dimensional. 
Proof. We repeat the proof of Theorem 1.4. The existence of a fixed point for $h$ follows from Theorem 1.1.

1.7. THEOREM. Let $\overline{\operatorname{coS}} \circ G: X \rightarrow C(X)$ be a compact mapping for a normal type II space $X$. Then $\overline{\cos } \circ G$ has a fixed point if $\overline{\cos } G(X)$ is finite dimensional.

Proof. Compare the proofs of Theorem 1.5 and Theorem 1.6.

2. Consequences of the fundamental theorems. The next four theorems are the immediate consequences, as every compact space is normal.

2.1. THEOREM. Let $G: X \rightarrow 2^{X}$ be an upper semicontinuous mapping for a compact type I space $X$. Then $F \circ G$ has a fixed point.

2.2. THEOREM. Let $\overline{\operatorname{coS}} \circ G: X \rightarrow C(X)$ be a compact mapping for a compact type I space $X$. Then $\overline{\operatorname{coS}} \circ G$ has a fixed point.

2.3. THEOREM. Let $G: X \rightarrow 2^{X}$ be a compact mapping for a compact type II space $X$. Then $F \circ G$ has a fixed point if $\overline{\cos } G(X)$ is finite dimensional.

2.4. THEOREM. Let $\overline{\operatorname{coS}} \circ G: X \rightarrow C(X)$ be a compact mapping for a compact type II space $X$. Then $\overline{\operatorname{coS}} \circ G$ has a fixed point if $\overline{\operatorname{coS}} G(X)$ is finite dimensional.

2.5. THEOREM. Let $X=\bar{X}$ be a normal type I subspace (for $S$ ) of an $S$-contractible space $Y$ and let $G: X \rightarrow 2^{Y}$ be such a mapping that $X \cap G$ and $(\overline{\operatorname{coS}} \circ(X \cap G))(X)$ are compact. Then $F \circ G$ has a fixed point.

Proof. In view of Theorem 1.4, $F_{\mid 2^{x}} \circ(X \cap G)$ has a fixed point. We have $\left(F_{12^{x}} \circ(X \cap G)\right)(x) \subset(F \circ G)(x)$ for $x \in X$ and therefore $F \circ G$ has a fixed point.

2.6. THEOREM. Let $X$ be a compact type I subspace (for $S$ ) of an $S$-contractible space $Y$ and let $G: X \rightarrow 2^{Y}$ be such a mapping that $X \cap G$ is compact. Then $F \circ G$ has a fixed point.

The analogs of the above two theorems for the type II spaces and for the function $\overline{\operatorname{coS}} \circ G$ can be deduced easily as $\overline{\cos } \circ(X \cap G)=X \cap \overline{(\cos } \circ(X \cap$ G)).

2.7. TheOREM. Let $X$ be a normal type I space and let $G: X \rightarrow T(X)$ be a compact mapping for which $\overline{\operatorname{coS}} G(X)$ is compact. Then $G$ has a fixed point.

Proof. The theorem is a consequence of Theorem 1.5 as $\overline{\operatorname{coS}} G(x)=G(x)$ for $x \in X$.

Theorem 2.7 is a generalization of Ky Fan's theorem for mappings in the locally convex spaces [4, Theorem 1].

2.8. Definition. A space $X$ is of type 0 (locally type 0 ), if it is $S$-contractible (locally $S$-contractible) for $S$ satisfying

for any $A \subset X$ and any neighborhood $V$ of $\cos A$ there exists a neighborhood $U$ of $A$ for which $\cos U \subset V$. 
It can be seen that every type 0 space is of type $I$.

2.9. Lemma. Let $\left\{X_{s}\right\}_{s \in T}$ be a family of type 0 spaces. Then $\Pi_{s \in T} X_{s}$ is of type 0 (similarly for the locally type 0 spaces).

Proof. Let it be $x=\Pi_{s \in T} x_{s}, y=\Pi_{s \in T} y_{s}$ and $t \in I$. Then $S_{x}(t, y):=\Pi_{s \in T} S_{x_{s}}^{s}\left(t, y_{s}\right)$ is the needed homotopy ( $S^{s}$ satisfy (9) for $s \in T$ ) because the projection is continuous $[3,2.3 .6$, p. 108] and the diagonal

$$
\Delta: \prod_{s \in T}\left(X_{s}^{X_{s}}\right)^{I} \rightarrow\left(\prod_{s \in T} X_{s}^{X_{s}}\right)^{I}
$$

is a homeomorphism. The other conditions can be easily checked.

2.10. LemMa. For an arbitrary set $A$ in a regular type 0 space we have $\overline{\cos } \boldsymbol{A}=$ $P_{A}:=\cap\{\overline{\cos } U: A \subset U=\operatorname{Int} U\}$.

Proof. Obviously $\overline{\operatorname{coS}} A \subset P_{A}$. Suppose $x \in P_{A}$ and $x \notin \overline{\cos } A$. There exists a neighborhood $V$ of $\overline{\operatorname{coS}} A$ for which $x \notin \bar{V}$. We can find a neighborhood $U$ of $A$ with $\overline{\operatorname{coS}} U \subset \bar{V}$, which gives a contradiction.

2.11. Corollary. For an arbitrary upper semicontinuous mapping $G: X \rightarrow 2^{X}$, $\overline{c o S} \circ G$ is upper semicontinuous, if $X$ is a regular type 0 space.

2.12. TheOREM. Let $G: X \rightarrow 2^{X}$ be a compact mapping for a normal type 0 space. Then $\overline{\operatorname{coS}} \circ G$ has a fixed point if $\overline{\operatorname{coS}} G(X)$ is compact.

Proof. This fact follows from Theorem 1.5 and 2.11.

2.13. TheOREM. Let $X$ be a compact type 0 space. Then for any compact $G$ : $X \rightarrow 2^{X}, \overline{\operatorname{coS}} \circ G$ has a fixed point.

We can easily formulate the type 0 versions of Theorems $2.5,2.6$.

Let us write for the nonempty subsets $A, D$ of a metric space $(M, d)$ and $x \in M$, $r>0$

$$
\begin{gathered}
d(x, A)=\inf \{d(x, y): y \in A\}, \quad d(A, D)=\inf \{d(x, D): x \in A\}, \\
B(A, r)=\{x \in M: d(x, A)<r\}, \quad P_{r}(D)=A \cap B(D, d(A, D)+r)
\end{gathered}
$$

and

$$
P(D)=\bigcap_{r>0} P_{r}(D)
$$

2.14. TheOREM. Let $A$ be a compact set of type $\mathrm{I}$ in a metric space $(M, d)$. Then $E \circ G:=\cap_{r>0} \overline{\operatorname{coS}}\left(P_{r} \circ G\right): A \rightarrow C(A)$ has a fixed point if $G: A \rightarrow C(M)$ is compact.

Proof. It is seen that $P \circ G: A \rightarrow C(A)$ and thus $\overline{\operatorname{coS}} P(G(A))$ is compact and $\left\{P_{r}(G(x))\right\}_{r>0}$ is a family of neighborhoods of $P(G(x))$ for which (4) holds with the suitable substitutions. In view of Theorem 1.4 it is enough to show that $P \circ G$ is upper semicontinuous.

There exist points $y \in G(x), z \in A \backslash B((P \circ G)(x), r)$ that give the distance between sets. Let us write $a_{r}=d(y, z)-d(A, G(x))$. Obviously $a_{r}>0$ and hence 
$P\left(B\left(G(x), a_{r} / 2\right)\right) \subset B((P \circ G)(x), r)$. Now it is seen that $P \circ G$ is upper semicontinuous.

If $G: A \rightarrow M$ is a map, $\overline{\cos } P_{r}(G(x))=A(G(x), r)$ and it is seen that Theorem 2.14 generalizes Theorem 4 from [10].

We have mentioned only two theorems for the type II spaces in the present section, but all the other theorems for the type I spaces in this paper can be easily transferred to the type II case.

\section{Generalized condensing and quasicompact mappings.}

3.1. Definition (CF. [6, pp. 12, 13]). Let $X$ be a space and for $\varnothing \neq Z \subset X$ let $G$ : $Z \rightarrow 2^{X}$ be a mapping. Then an $S$-contractible set $D=\bar{D} \subset X$ is characteristic of $G$ if $Z \cap D \neq \varnothing, G(Z \cap D) \subset D$ and $\cos G(Z \cap D)$ is compact (in the case $G=\overline{c o S} \circ H$ we assume only the compactness of $\overline{G(Z \cap D)}$ ).

Let $X$ be an $S$-contractible space and $W=\overline{\operatorname{coS}} W \subset X, K=\overline{\cos } K \subset X$; a mapping $G: W \cap K \rightarrow 2^{K}$ is quasicompact if it has a characteristic set on which $G$ is upper semicontinuous.

3.2. TheOREM. Let $X$ be a normal type I space for which $\overline{\operatorname{coS}} \circ G: X \rightarrow C(X)$ is quasicompact. Then $\overline{c o S} \circ G$ has a fixed point.

Proof. See Theorem 1.5.

3.3. THEOREM. Let $X$ be a normal type I space for which $G: X \rightarrow 2^{X}$ is quasicompact. Then $F \circ G$ has a fixed point.

Proof. Let $D$ be a set characteristic of $G \neq \overline{\operatorname{coS}}$ ○ $H$. Then from Theorem 1.4 follows the existence of $x_{0} \in\left(F_{\mid 2^{D}} \circ G\right)\left(x_{0}\right) \subset(F \circ G)\left(x_{0}\right)$. If $G=\overline{\operatorname{co}} S \circ H, G$ itself has a fixed point (Theorem 3.2) and always $G(x) \subset(F \circ G)(x)$.

3.4. Definition (CF. [2], [6, p. 18]). Let $X$ be an $S$-contractible space and $\varnothing \neq Z \subset X$. Then $G: Z \rightarrow 2^{X}$ is generalized condensing if it is upper semicontinuous for compact $Q$ with $G(Q) \subset Q$ and

$$
\begin{aligned}
& \text { for any } Q \subset Z \text { with } G(Q) \subset Q, \operatorname{card}(Q \backslash \overline{G(Q)})<1 \text { implies } \\
& \overline{G(Q)} \text { is compact, }
\end{aligned}
$$$$
Q \subset Z, Q=\overline{\cos } G(Q) \text { imply the compactness of } Q \text {. }
$$

3.5. Definition [8]. A space $X$ is $\bar{S}$-contractible if it is $S$-contractible and $\overline{\operatorname{co}} S A$ is $S$-convex for any $A \subset X$.

3.6. Definition [8]. A space $X$ is of type $\overline{\mathrm{I}}$ (type $\overline{\mathrm{II}}$ ) provided that it is $\bar{S}$-contractible and of type I (type II) for $S$.

The next lemma was proved in [8] (cf. [9]).

LEMMA. If $G: X \rightarrow 2^{X}$ is such a mapping for $\bar{S}$-contractible space $X$ for which there exists a compact set $B \supset G(B)$, there exists a set $D=\overline{\operatorname{coS}} D=\overline{\operatorname{co}} S G(D) \neq \varnothing$.

For the locally convex spaces it is known that every generalized condensing mapping is quasicompact $[6,1.3 .8$, p. 18]. We obtain here a similar result. 
3.7. TheOREM. Let $G: X \rightarrow 2^{X}$ be a generalized condensing mapping for a type $\overline{\mathrm{I}}$ space. Then $F \circ G$ has a fixed point.

Proof. It is enough to show that $G$ has a compact characteristic set (cf. [2, Theorem 2, p. 129]).

Let $x \in X$ be arbitrary. Assume $B=\bar{B}$ to be a minimal set containing $x$ with the property $G(B) \subset B$. It can be seen that $B \backslash \overline{G(B)} \subset\{x\}$ because $(B \backslash \overline{G(B)}) \cap$ $(X \backslash\{x\})$ is open in $B$ and would be rejected while being nonempty. In view of (10) and lemma there exists a nonempty set $D=\overline{\operatorname{coS}} G(D)$, which is compact (see (11)).

We can easily obtain an analog of Theorem 3.7 for the type $\overline{\mathrm{I}}$ spaces.

\section{Minimax theorem.}

4.1. Lemma (CF. [5]). Let $X$ be an $S$-contractible subspace of a space $Y$. Suppose $G$ : $X \rightarrow C(Y)$ satisfies

$$
\begin{aligned}
& \left\{x_{1}, \ldots, x_{n}\right\} \subset X \text { implies for } n \in N \\
& S_{x_{1}}\left(I, S_{x_{2}}\left(I, \ldots, S_{x_{n-1}}\left(I, x_{n}\right) \ldots\right) \subset \cup_{n}^{i=1} G\left(x_{i}\right),\right.
\end{aligned}
$$

Then $\cap_{x \in X} G(x) \neq \varnothing$.

$$
\text { for at least one } x \in X, G(x) \text { is compact. }
$$

Proof (CF. [5]). It is enough to prove that $\cap_{i=1}^{n} G\left(x_{i}\right) \neq \varnothing$ for any $n \in N$ and $x_{1}, \ldots, x_{n} \in X\left[3,3.1 .1\right.$, p. 166]. Let us consider $G_{i}:=g^{-1}\left(G\left(x_{i}\right)\right)$ (see (2)). It follows from (12) that $\cap_{i=1}^{n} G_{i} \neq \varnothing[3$, Theorem 4, p. 510].

REMARK. Instead of (12) we can use the following stronger but more elegant condition: $x_{1}, \ldots, x_{n} \in X$ implies $\operatorname{coS}\left\{x_{1}, \ldots, x_{n}\right\} \subset \cup_{i=1}^{n} G\left(x_{i}\right)$ for any $n \in N$ and $x_{i} \in X$.

4.2. Lemma (CF. [5]). Let $X$ be an $S$-contractible space and let $A \subset X \times X$ be compact. Assume

(i) $(x, x) \in A$ for $x \in X$,

(ii) $\{x:(x, y) \notin A\}$ is $S$-convex for $y \in X$.

Then there exists $y_{0} \in X$ for which $X \times\left\{y_{0}\right\} \subset A$.

Proof (CF. [5]). Let it be $G(x):=\{y \in X:(x, y) \in A\}$ for $x \in X$. Suppose there exists some $x$ with

$$
S_{x_{1}}\left(t_{1}, \ldots, S_{x_{n-1}}\left(t_{n-1}, x_{n}\right) \ldots\right)=x \notin \bigcup_{i=1}^{n} G\left(x_{i}\right) .
$$

It follows from the definition of $G$ that $\left(x_{i}, x\right) \notin A$ for $i=1, \ldots, n$ and in view of (ii) $(x, x) \notin A$ which contradicts (i). Lemma 4.1 guarantees the existence of $y_{0} \in \bigcap_{x \in X} G(x)$ means $\left(x, y_{0}\right) \in A, x \in X$.

4.3. LemMa (CF. [7]). Let $X_{1}, X_{2}$ be compact type I spaces for $S_{1}, S_{2}$ respectively. Assume $U, V$ are closed subsets of $X_{1} \times X_{2}$ and $\varnothing \neq U_{x}:=\left\{y \in X_{2}:(x, y) \in U\right\}$ $=\overline{\operatorname{coS}}_{2} U_{x}, \varnothing \neq V_{y}=\left\{x \in X_{1}:(x, y) \in V\right\}=\overline{\operatorname{coS}}_{1} V_{y}$. Then we have $U \cap V \neq \varnothing$. 
Proof (cf. [7]). We will prove for example that $\left\{V_{y}\right\}$ is a compact mapping. Let $W_{y}$ be a neighborhood of $V_{y}$. Suppose that for every neighborhood $Z$ of $y$ there exists a point $\left(x_{z}, z\right) \in V_{z} \times Z$ that does not belong to $W_{y} \times Z$. A net with the values $\left(x_{z}, z\right)$ has a cluster point of the form $(x, y) \in V[3,3.1 .23$, p. 172] which contradicts $(x, y) \notin V_{y} \times\{y\}$.

The mapping $G: X_{1} \times X_{2} \rightarrow T\left(X_{1} \times X_{2}\right)$ with the values $G(x, y):=V_{y} \times U_{x}$ (cf. Lemma 2.9) is compact as being upper semicontinuous and has a fixed point (Theorem 2.7). Let it be $\left(x_{0}, y_{0}\right) \in V_{y_{0}} \times U_{x_{0}}$. Then we have $x_{0} \in V_{y_{0}}, y_{0} \in U_{x_{0}}$ which means $\left(x_{0}, y_{0}\right) \in U \cap V$.

4.4. TheOREM (CF. [4], [7]). Let $f: X_{1} \times X_{2} \rightarrow R$ be such a map for the compact type I spaces $X_{1}, X_{2}$, that for $p, q \in R$

$$
\begin{aligned}
& U_{x}^{q}:=\left\{y \in X_{2}: f(x, y)<q\right\}=\overline{\cos } U_{2} U_{x}^{q} \\
& V_{y}^{p}:=\left\{x \in X_{1}: f(x, y) \geqslant p\right\}=\overline{\operatorname{coS}} V_{1} V_{y}^{p} .
\end{aligned}
$$

Then

$$
\max _{x \in X_{1}} \min _{y \in X_{2}} f(x, y)=\min _{y \in X_{2}} \max _{x \in X_{1}} f(x, y) .
$$

Proof [7]. Take

$$
\begin{gathered}
U=\left\{z_{0}=\left(x_{0}, y_{0}\right): f\left(x_{0}, y_{0}\right) \leqslant \min _{y \in X_{2}} f\left(x_{0}, y\right)\right\}, \\
V=\left\{z_{0}: f\left(x_{0}, y_{0}\right)>\max _{x \in X_{1}} f\left(x, y_{0}\right)\right\} .
\end{gathered}
$$

In view of Lemma 4.3, $U \cap V \neq \varnothing$. So there exists $z_{0}$ with

$$
f\left(x_{0}, y_{0}\right)=\min _{y \in X_{2}} f\left(x_{0}, y\right)=\max _{x \in X_{1}} f\left(x, y_{0}\right) \text {. }
$$

Hence we obtain

$$
\begin{aligned}
\min _{y \in X_{2}} \max _{x \in X_{1}} f(x, y) & \leqslant \max _{x \in X_{1}} f\left(x, y_{0}\right)=f\left(x_{0}, y_{0}\right) \\
& =\min _{y \in X_{2}} f\left(x_{0}, y\right)<\max _{x \in X_{1}} \min _{y \in X_{2}} f(x, y) .
\end{aligned}
$$

The theorem is proved as obviously we have

$$
\min _{y \in X_{2}} \max _{x \in X_{1}} f(x, y) \geqslant \max _{x \in X_{1}} \min _{y \in X_{2}} f(x, y) .
$$

\section{REFERENCES}

1. C. Berge, Espaces topologiques, fonctions multivoques, Dunod, Paris, 1966.

2. J. Danes, Generalized concentrative mappings and their fixed points, Comm. Math. Univ. Carolinae 11 (1970), 115-136.

3. R. Engelking, General topology, PWN, Warsaw, 1977.

4. K. Fan, Fixed-point and minimax theorems in locally convex topological linear spaces, Proc. Nat. Acad. Sci. U.S.A. 38 (1952), 121-126.

5. __, A generalization of Tychonoff's fixed point theorem, Math. Ann. 142 (1961), 305-310. 
6. S. Hahn, Zur Theorie nichtlinearer Operatorengleichungen in topologischen Vektorräumen, Thesis, Dresden, 1977.

7. S. Kakutani, A generalization of Brouwer's fixed point theorem, Duke Math. J. 8 (1941), 457-459.

8. L. Pasicki, A generalization of Reich's fixed point theorem, Comment. Math. 23 (to appear).

9. __, On the measures of noncompactness, Comment. Math. 21 (1979), 203-205.

10. __ Retracts in metric spaces, Proc. Amer. Math. Soc. 78 (1980), 595-600.

11. _ Three fixed point theorems, Bull. Acad. Polon. Sci. 28 (1980).

Department of Mathematics, Science School of Mining and Metallurgy, Kraków, Poland

Current address: Dzierzynskiego 115/44, 30-058 Kraków, Poland 